\title{
Evaluation of the Use of Wetlands in Arsenic Sequestration of Mine-Influenced Effluent using Synchrotron XRF and XANES Spectroscopy.
}

\author{
Connor Arduini ${ }^{1}$, Lauchlan Fraser ${ }^{1}$, Trevor Flynn², Lisa Van Loon ${ }^{2,3}$, and Neil Banerjee ${ }^{2 *}$ \\ 1. Faculty of Science, Thompson Rivers University, Kamloops, Canada. \\ 2. Department of Earth Sciences, Western University, Canada. \\ 3. LISA CAN Analytical Solutions Inc., Saskatoon, Canada. \\ * Corresponding author, neil.banerjee@uwo.ca
}

As a resource-driven economy, it is important for Canada to encourage environmental protection and sustainability in the mining industry if it is to maintain its status as a global mining leader. In 2015, British Columbia's mining industry was valued at $\$ 5.9$ billion, approximately $13.8 \%$ of the GDP of the province [1]. As such, BC is an integral contributor to Canada's mining industry. Currently, there are 24 mineral and coal mines operating in $\mathrm{BC}$, with several others proposed or undergoing development. The $\mathrm{BC}$ Mines Act provides legislation regarding how mining activities are carried out and includes guidelines for reclamation, which is the process of returning disturbed land into a functional ecosystem.

During monitoring of mine tailings water at one mine site, it has been determined that arsenic is the major contaminant of concern that will require treatment following mine closure. Arsenic is found in the wet mine tailings storage facility (TSF), and a pilot project involving a passive bioreactor at the toe of the TSF is currently underway. In addition, a natural seep periodically releases arsenic-contaminated water into a natural wetland located on the mine site. Preliminary tests of water inflow and outflow show that arsenic is sequestered within the wetland and is not detectable in the effluent. The wetland provides a unique opportunity to test the natural biogeochemical processes and pathways of arsenic through this system and to determine causal factors that lead to sequestration of this potentially harmful element. This study builds a collaboration between experts in ecosystem reclamation, passive treatment wetlands, and in synchrotron science to evaluate arsenic treatments in mine wastes.

By using synchrotron radiation X-ray fluorescence (SR-XRF) analysis, the presence and occurrence of arsenic species and other potentially deleterious elements can be resolved (Figure. 1). Using X-ray absorption near edge structure (XANES) analysis, the oxidation state of arsenic can be elucidated, providing information on redox states as well as the nature of any arsenic species present (Figure. 1). Ultimately, this project will develop an understanding of the occurrence of arsenic and more importantly its chemical speciation in samples from the wetland. This information will help characterize the mechanisms of arsenic capture, the likelihood for long term sequestration of arsenic, and the ability of the wetland to provide passive arsenic removal.

Experimental: Samples consisted of soil, roots, and leaves collected in the wetland, at three distances from the water inflow location to determine differences in arsenic abundance and speciation. SR-XRF data was collected at CLS@APS: 20-ID using an incident energy of $26 \mathrm{keV}$ with an unfocussed beam spot size of $600 \mu \mathrm{m} \times 600 \mu \mathrm{m}$ and a $90 \mathrm{sec}$ dwell time. SR-XRF data was collected for three spots on each sample to determine homogeneity of the samples. Soil samples were prepared as homogenized pellets and the leaves and roots were left as cut samples. All samples were attached to a holder with Kapton Tape and covered with $6 \mu \mathrm{m}$ Mylar. Arsenic K-edge XANES data was collected in fluorescence mode for the soil and root samples with the detector windowed to correspond to the As Ka fluorescence 
emission peak to maximize the signal to noise. Data were collected over a relative energy range of -150 $\mathrm{eV}$ to $9 \mathrm{k}$, with a dwell time of $1 \mathrm{sec}$ per point and a step size of $0.5 \mathrm{eV}$ near the edge.

Two-dimensional SR- $\mu$ XRF maps and $\mu$-XANES analyses were collected at the VESPERS beamline at the CLS. SR- $\mu$ XRF mapping of the soils, roots, and leaves used pink beam conditions and a 4-element SDD (Figure. 1.). Filters were used if necessary, to keep the detector dead time at $<25 \%$. XRF maps were collected using a $5 \mu \mathrm{m}$ x $5 \mu \mathrm{m}$ spot size and a dwell time of $1 \mathrm{sec}$ per point (Figure. 1.; left).

Arsenic K-edge $\mu$ XANES data was collected at As hotspots (Figure. 1.; right) identified in the SR- $\mu$ XRF maps. As K-edge XANES data was collected in fluorescence mode with the detector windowed to correspond to the As Ka fluorescence emission peak to maximize the signal to noise. The detector was moved in to a distance of $50 \mathrm{~mm}$ from the sample to increase the signal. Data were collected over a relative energy range of $-150 \mathrm{eV}$ to $8 \mathrm{k}$, with a dwell time of $1 \mathrm{sec}$ per point and a step size of $0.5 \mathrm{eV}$ near the edge. All XRF data were analyzed using the Peakaboo software package, developed at Western University [2]. XANES data were analyzed using the Athena software program [3].

The preliminary results show a decrease in the arsenic abundance as a function of distance from the water inflow. Differences are also observed between arsenic abundance in the soils, roots, and leaves, with very little arsenic detected in the leaves for all three locations. Differences are observed in arsenic speciation between the soil samples, while As speciation in the roots samples is similar. The As concentration was below detection limits for XANES analysis in the leaves. Further analysis is ongoing to better spatially evaluate the passive wetland treatment of arsenic-containing mine-influenced effluent $[4]$.

\section{References:}

[1] Mining Association of Canada, www.mining.ca (2016).

[2] N. Sherry et al, Science Studio Project Summary. (2012) http://sciencestudio.net/summary.php.

[3] B. Ravel, M. Newville. J. Synchr. Rad. 12 (2005) p. 537.

[4] Research described in this paper was performed at the Canadian Light Source (CLS) and Advanced Photon Source (APS). The CLS is supported by CFI, NSERC, the University of Saskatchewan, the Government of Saskatchewan, Western Economic Diversification Canada, the NRC, and CIHR. The APS is an Office of Science User Facility operated for the U.S. Department of Energy (DOE) Office of Science by Argonne National Laboratory, and is supported by the U.S. DOE under Contract No. DEAC02-06CH11357. The authors thank Renfei Feng, Peter Blanchard, and Zou Finfrock for their support in conducting the experiments. The authors acknowledge funding from NSERC.
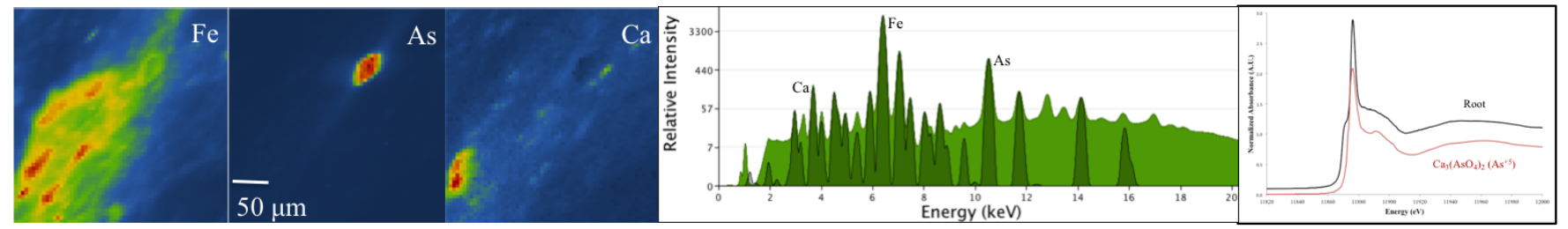

Figure. 1. 2D SR- $\mu \mathrm{XRF}$ maps (left) of $\mathrm{Fe}, \mathrm{As}$, and $\mathrm{Ca}$ in a root sample. Each pixel contains a complete XRF MCA spectrum (middle). As K-edge $\mu$ XANES spectrum (right) was collected at the arsenic hotspot. Comparison with standards indicates that $\mathrm{As}$ is a $\mathrm{As}^{+5}$ arsenate. 